\title{
MULTIPLICATIVE PERTURBATION BOUNDS FOR WEIGHTED UNITARY POLAR FACTOR
}

\author{
Hu YANG, HANYU Li AND HuA SHAO
}

\begin{abstract}
The multiplicative perturbation bounds for weighted unitary polar factor are considered in the weighted unitary invariant norm, weighted spectral norm, and weighted Frobenius norm in this paper. As the special cases, new bounds for subunitary and unitary polar factor are also derived. These new bounds improve the corresponding results published recently to some extent.
\end{abstract}

Mathematics subject classification (2010): 15A45, 65F35.

Keywords and phrases: Weighted polar decomposition, weighted unitary polar factor, singular value decomposition, perturbation bound.

\section{REFERENCES}

[1] A. Ben-Israel and T. N. E. Greville, Generalized Inverses: Theory and Applications. 2nd ed., Springer, New York, 2003.

[2] X.S. Chen AND W. Li, Perturbation bounds on the polar decomposition under unitarily invariant norms, Math. Numer. Sin., 27 (2005), 121-128.

[3] X.S. Chen AND W. LI, Relative perturbation bounds for subunitary polar factor under unitarily norm, Adv. Math. (China), 35 (2006), 178-184.

[4] X.S. Chen AND W. LI, Variations for $Q$ - and $H$-fators in the polar decomposition, Calcolo, 45 (2008), 99-109.

[5] X.S. Chen, W. LI AND W. Sun, Some new perturbation bounds for the generalized polar decomposition, BIT, 44 (2004), 237-244.

[6] C. DAVIS AND W. M. KAHAN, The rotation of eigenvectors by a perturbation, III. SIAM J. Numer. Anal., 7 (1970), 1-46.

[7] N.J. Higham, Computing the polar decomposition—with applications, SIAM J. Sci. Statist. Comput., 7 (1986), 1160-1174.

[8] R.C. LI, A perturbation bound for the generalized polar decomposition, BIT, 33 (1993), 304-308.

[9] R.C. LI, New perturbation bounds for the unitary polar factor, SIAM J. Matrix Anal. Appl., 16 (1995), 327-332.

[10] R.C. LI, Relative perturbation bounds for the unitary polar factor, BIT, 37 (1997), 67-75.

[11] R.C. LI, Relative perturbation theory: (II). Eigenspace and singular subspace variations, SIAM J. Matrix Anal. Appl., 20 (1998), 471-492.

[12] R.C. LI, Relative perturbation bounds for positive polar factors of graded matrices, SIAM J. Matrix Anal. Appl., 27 (2005), 424-433.

[13] W. LI, On the perturbation bound in unitarily invariant norms for subunitary polar factors, Linear Algebra Appl., 429 (2008), 649-657.

[14] W. LI, Some new perturbation bounds for subunitary polar factors, Acta Math. Sin., 21 (2005), 15151520.

[15] W. Li And W. Sun, Combined perturbation bounds: II. Polar decompositions, Sci. China Ser. A, 50 (2007), 1339-1346.

[16] W. Li AND W. Sun, Perturbation bounds of unitary and subunitary polar factors, SIAM J. Matrix Anal. Appl., 23 (2002), 1183-1193. 
[17] W. LI AND W. SUN, New perturbation bounds for unitary polar factors, SIAM J. Matrix Anal. Appl., 25 (2003), 362-372.

[18] W. LI AND W. SUN, Some remarks on the perturbation of polar decompositions for rectangular matrices, Numer. Linear Algebra Appl., 13 (2005), 327-338.

[19] C. R. RAo AND S. K. Mitra, Generalized Inverses of Matrices and its Applications, Wiley, New York, 1971.

[20] C. R. Rao And M. B. RaO, Matrix Algebra and its Applications to Statistics and Econometrics, World Scientific, Hong Kong, 1998.

[21] J.G. Sun AND C.H. Chen, Generalized polar decomposition, Math. Numer. Sin., 11 (1989), 262 273.

[22] C. F. VAn LOAN, Generalizing the singular value decomposition, SIAM J. Numer. Anal., 13 (1976), $76-83$.

[23] G.R. WAng, Y.M. WeI AND S.Z. QIAO, Generalized Inverses: Theory and Computations, Science Press, Beijing, 2004.

[24] H. YANG AND H.Y. LI, Perturbation bounds for weighted polar decomposition in the weighted unitarily invariant norm, Numer. Linear Algebra Appl., 15 (2008), 685-700.

[25] H. YANG AND H.Y. LI, Weighted polar decomposition, J. Math. Res. Exposition, in press.

[26] H. YANG AND H.Y. LI, Weighted polar decomposition and WGL partial ordering of rectangular complex matrices, SIAM J. Matrix Anal. Appl., 30 (2008), 898-924.

[27] H. YANG AND H.Y. LI, Weighted UDV*-decomposition and weighted spectral decomposition for rectangular matrices and their applications, Appl. Math. Comput., 198 (2008), 150-162. 